\title{
Anterior Surgery in Multilevel Stenosis of the Lower Cervical Spine: Technical Indications and Personal Experience. 12 Years Follow-Up
}

\author{
Alessandro Landi', Nicola Marotta, Cristina Mancarella, Carlotta Morselli, Roberto Tarantino, \\ Andrea Ruggeri, Roberto Delfini
}

Department of Neurology and Psychiatry, Division of Neurosurgery, University of Rome "Sapienza”, Roma, Italy. Email: *dott.alessandro.landi@gmail.com

Received January $7^{\text {th }}, 2014$; revised January 31 ${ }^{\text {st }}$,2014; accepted February $15^{\text {th }}, 2014$

Copyright (C) 2014 Alessandro Landi et al. This is an open access article distributed under the Creative Commons Attribution License, which permits unrestricted use, distribution, and reproduction in any medium, provided the original work is properly cited. In accordance of the Creative Commons Attribution License all Copyrights (C) 2014 are reserved for SCIRP and the owner of the intellectual property Alessandro Landi et al. All Copyright (C) 2014 are guarded by law and by SCIRP as a guardian.

\begin{abstract}
Objective: cervical spondylotic myelopathy is a progressive degenerative cervical spine disease. During later stages of segmental degeneration, kyphosis of the cervical spine can occur and further compromise the spinal cord and nerve roots. Optimal surgical approach remains controversial. The choice to perform an anterior, posterior or combined approach depends on: sagittal alignment, number of involved levels, main compression localization, and clinical status. The anterior approach is recommended when compression involves primarily anterior horn of spinal cord. Methods: between January 2001 and December 2005, 121 patients (42 F, 79 M, mean age 62 years) were operated for cervical spondylosis (98 myelopathy, 23 radiculopathy). Anterior surgical approach was performed in 81 patients. 63 patients were operated performing multilevel discectomy and fusion (ACDF) and 18 patients performing corpectomy and fusion and anterior plating (ACCF). Preoperative documentation collected consisted of cervical X-ray (static-dynamic), cervical spine TC, cervical MRI. Clinical documentation permitted us to obtained clinical status of each patient based on JOA, NDI and VAS. A Clinical and radiological follow-up was performed at 1 month, 3 months, 1 year, 6 years, 12 years. Results: the fusion rate was calculated based on the static and dynamic $X$-ray (flexion and extension position), only a little percentage of patients underwent CT scan. There were no significant differences between ACDF and ACCF in clinical outcome at 6 years evaluated by VAS and NDI. The rate of fusion at 6 years for 2 levels ACCF (92\%) was higher than that for 2 levels ACDF $(86 \%)$ but is not statistically significative. Conclusion: classifying degenerative disease and biomechanics feature, preoperatively in necessary to guide the surgeon to choose the best anterior approach for cervical spondylosis.
\end{abstract}

\section{KEYWORDS}

Corpectomy; Discectomy; Multilevel; Cervical Spine

\section{Introduction}

Degenerative cervical spine can be managed effectively with conservative (nonsurgical) treatment, however, severe multilevel spondylosis may require surgical attention if symptoms persist through several weeks of conservative treatment [1].

Cervical spine degeneration can be caused by degenerative disk disease, vertebral body instability, trauma

"Corresponding author. or post-traumatic deformity, tumors or even infections. Cervical spondylotic myelopathy (CSM) [2] is a progressive degenerative cervical spine disease that can evolve in some main different stages: the disc height decreases leading to disc bulging as a result of progressive changes to the extracellular matrix of the disc. Microinstability results in reactive hyperostosis with formation of osteophytes at the vertebral endplates which can penetrate into the spinal canal and compromise the spinal cord and nerve roots. Osteophytes of the uncovertebral and facet 
joints reduce the mobility of the segment. Segmental instability leads to a hypertrophy of the yellow ligament and causes a narrowing of the spinal canal and foramen. During later stages of segmental degeneration, kyphosis of the cervical spine can occur and further compromise the spinal cord and nerve roots [1]. Optimal surgical approach remains controversial.

The choice to perform an anterior, posterior o combined approach depends on sagittal alignment, number of involved levels, main compression localization, and clinical status [3-6]. In case of cervical myeloradiculopaty with multiple levels involved (>3), developmental stenosis, posterior compression or in case of OPLL, posterior procedure may be preferable [7].

The anterior approach is much safer and not much traumatic for muscles and familiar to the surgeon. It is recommended when compression involves primarily anterior horn of spinal cord. The main goal of this procedure is to relieve the compression on the spinal cord and/or the root. Stabilization and fusion in conjunction with decompression are important to hinder the progress of phenomena such as instability and subsequent deformity of the cervical segment, and to restore the height, correct the in-buckling of the ligamentum flavum, recostruct lordosis, and stabilize the spinal columm $[7,8]$.

\section{Materials and Methods}

Between January 2001 and December 2005, 121 patients (42 F, $79 \mathrm{M}$, mean age 62 years) were operated in the Department of Neurological and Neurosurgical Sciences of Rome "Sapienza" for cervical spondylosis (98 myelopathy, 23 radiculopathy). Anterior surgical approach was performed in 81 patients, 4 underwent combined surgical approach and 36 posterior surgical approach.

Our study includes the 81 patients that underwent anterior surgical approach (51 males, 30 females, mean age 58 years). Inclusion criteria were:

1) multi-level cervical stenosis involving up to 3 levels (1 to 3 levels);

2) presence of signs and symptoms of cervical myelopathy;

3) presence of osteophytes and disc herniation;

4) radiological signs of instability;

5) cervical kyphosis.

Exclusion criteria included:

1) involvement of more than 3 levels;

2) OPLL;

3) instability or stenosis in patients affected by chronic diseases (diabetes mellitus, AR, ankylosing spondylitis) for which we performed a combined approach.

The patients included in the study were divided retrospectively into two main groups according to the procedure used: patient operated performing ACDF, 53 at 2 levels and 10 at 3 levels (mean age 58 years, $25 \mathrm{~F}$ and 38 M) (Multilevel discectomy and fusion) and patients operated performing ACCF, 14 at 2 levels and 4 at 3 levels (mean age 57 years, $5 \mathrm{~F}$ and $13 \mathrm{M}$ ) (corpectomy and fusion and anterior plating).

Preoperative documentation of each patient has been collected. It consisted of cervical X-ray (static-dynamic), cervical spine TC, cervical MRI. Clinical documentation permitted us to obtain clinical status of each patient based on JOA, NDI and VAS. A Clinical and radiological follow-up was performed at 1 month, 3 months, 1 year, 6 years, 12 years.

In accordance with the recent literature and from the analysis of data both 2 groups were evaluated and compared, evaluating preoperative clinical status (JOA, NDI, VAS), preoperative radiological exams (site of compression), number of levels involved, the state of the LLP: normal, segmental ossification, multi-level hypertrophy or ossification, osteophytosis, straightening of the cervical spine, bone characteristics, degree of stability/instability, associated with cervical hernia. The aim of this study is to evaluate the clinical and radiological outcome to help us to make the right choice between the two types intervention.

\section{Results}

The fusion rate was calculated based on the static and dynamic X-ray (flexion and extension position), only a little percentage of patients underwent CT scan. In the patients who underwent 2-level ACDF (total 53 patients) 46 showed fusion rate at 6 years of $86 \%$ (Figure 1) and in the remaining 7 it documented by the $\mathrm{X}$-ray, pseudoarthrosis. For patients who underwent 3 levels ACDF (10 patients), 6 showed a good fusion rate (60\%), instead 4 documented pseudoarthrosis (Table 1). In the patients who underwent 2-level ACCF (14 patients) 13 showed fusion rate at 6 years of 92\% (Figure 2) and 1 documented pseudoarthrosis. For patients who underwent 3 levels ACCF (4 patients), 3 showed a good fusion rate (75\%), instead 1 documented pseudoarthrosis (Table 2).

2 patients who underwent ACCF required a second operation because of the worsening of the clinical status due to the failure in correcting the kyphosis. In those patients was placed an expansion mesh restoring sagittal alignment.

For clinical assessment of patients, two scales were employed: VAS and NDI. The VAS score for neck pain in the patients who underwent multiple discectomy decreased significantly from 8 pre-operatively to 3 postoperatively at 3 and 6 months and then slightly increased during the follow-up to 4 . There was also an improvement of the NDI from 35\% pre operatively to $15 \%$ - 16\% post-operatively respectively after 3 and 6 months and 


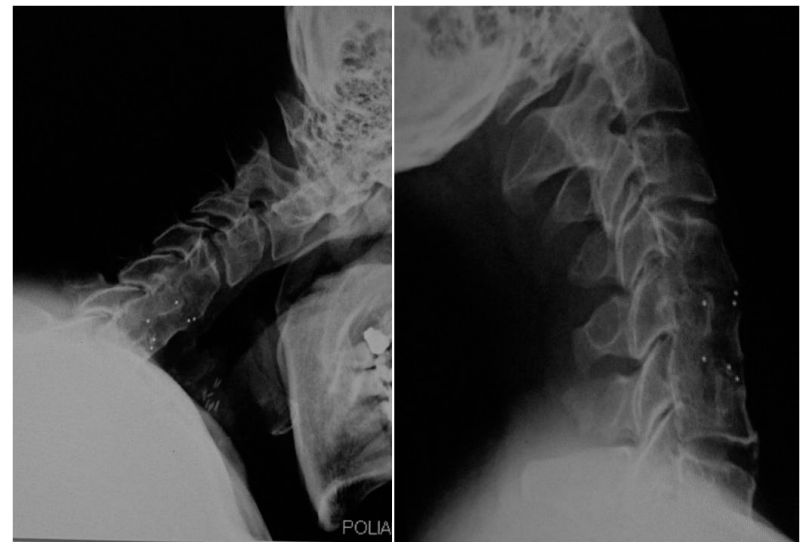

Figure 1. Dynamic cervical spine $\mathrm{X}$-ray in a patient who underwent 2 level discectomy.

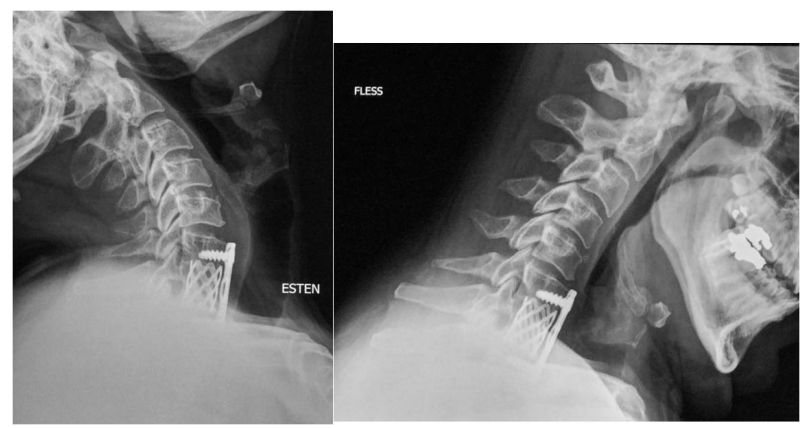

Figure 2. Dynamic cervical spine X-ray in patient who underwent 2 level corpectomy.

Table 1. Rate of fusion evacuated with $\mathrm{X}$-ray in patients treated with ACDF.

\begin{tabular}{ccccc}
\hline Discectomy & & & & \\
\hline Level Treated & $\begin{array}{c}\text { No. of } \\
\text { Patients }\end{array}$ & Fusion & Pseudarthrosis & $\begin{array}{c}\text { Fusion Rate } \\
\text { (\%) }\end{array}$ \\
\hline 2 Levels & 53 & 46 & 7 & $86 \%$ \\
\hline 3 Levels & 10 & 6 & 4 & $60 \%$ \\
\hline
\end{tabular}

Table 2. Rate of fusion evacuated with $\mathrm{X}$-ray in patients treated with ACCF.

\begin{tabular}{ccccc}
\hline Corpectomy & & & & \\
\hline Level Treated & $\begin{array}{c}\text { No. of } \\
\text { Patients }\end{array}$ & Fusion & Pseudarthrosis & $\begin{array}{c}\text { Fusion Rate } \\
\text { (\%) }\end{array}$ \\
\hline 2 Levels & 14 & 13 & 1 & $92 \%$ \\
\hline 3 Levels & 4 & 3 & 1 & $75 \%$ \\
\hline
\end{tabular}

$21 \%$ at the latest follow-up. The Vas score for the patients who underwent anterior corpectomy decreased from 7.7 pre-operatively to 4.1 - 3.9 post-operatively at 3 and 6 months and then increased during the follow-up to 4. The improvement of the NDI was from 38\% pre operatively to $11 \%$ post-operatively after 3 and 6 months and
$21 \%$ at the latest follow-up. Mean VAS and NDI score were statistically different between preoperation and post operation in each group. VAS and NDI score were not significantly different in both ACDF and ACCF in the immediate postoperatory ( $<<0.05$ ). The scores in the ACDF were significantly higher compared to that in the ACCF group $(p=0.001)$ after 6 years from the surgery (Figure 3).

\section{Discussion}

The choice between ACDF or ACCF as the best treatment of multilevel cervical spodylosis is object of numerous studies, but actually remains controversial [9-12].

It is argued that cervical spine compression due to a degeneration process tends to be progressive. In fact, it is the results of disc degeneration which leads to the reduction of the height disc, to hypertrophy and buckling of the ligamentum flavum and PLL, osteophytes formation, alterations in axial loads with sagittal alignment disfuction. The degenerative cascade brings to a progressive canal narrowing, signs and symptoms of myelin dysfunction and radiological finding of cervical instabilit]y [1,2]. Often even if asymptomatic at first, radiological degeneration can be shown at the radiological exams. Sometime the evolution could be quite progressive (JOA $>12$, or age $<75$, Class 1 ) and poor or not symptomatic, but sometime certain patients (20\%) may have a precipitous decline with clinically and radiological severe myelopathy [13]. The goals of surgical treatment are: to decompress the stenotic cervical segment, to arrest the degenerative process, and to restore the physiological biomechanics and the right axial loads.

In our opinion it is important to consider some radiological preoperative findings which may lead to the right indication for surgery.

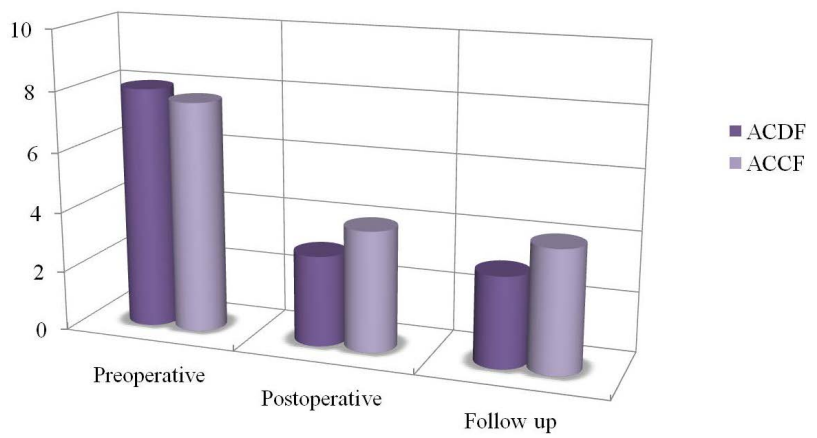

Figure 3. Clinical outcome of patients treated with ACCF and ACDF. Mean VAS and NDI score were statistically different between preoperation and post operation in each group. The scores in the ACDF were significantly higher compared to that in the ACCF group $(p=0.001)$ after 6 years from the surgery. 
1) Number of involved levels: when 3 or less levels are involved, anterior compression or kyphosis are present, the anterior approach is preferred. Anterior approach allows direct decompression, interbody space hight restore and cervical lordosis maintenance. Several literature studies have compared the multilevel anterior cervical discetomy and fusion (ACDF) with the anterior cervical corpectomy and fusion (with mesh cage) (ACCF), but the right surgical procedure remain controversal.

2) Radiological and neurological signs: radiological exams as like as standard X-ray in the anterioposterior and lateral projections and the dynamic flexion and extension projections are important to value: sagittal profile, as loss of lordosis, kyphosis development, spinal alignment and bony relationship (e.g. spondylolisthesis), disc space narrowing, bony vertebral structures (vertebral collapse, osteophytes).

3) LLP features (hyperthrophy, ossification, involved levels): in multilevel cervical spondylosis may occur involvement of the LLP. It can be the responsible for a further reduction of the medullar canal, because of its involvement in the degenerative cascade of spondylodiscoarthosis. LLP modification occurs, in primis, with structural modifications caused by local inflammatory processes that lead than to an initial ligamentous laxity. LLP undergo than to a compensatory hypertrophy and subsequent ossification. The involvement of the ligament reflects the number of levels involved in the spondylodiscoarthosis.

4) Vertebral stability: static and dynamic radiographs are useful to determine the range of motion (ROM) of the cervical segment and to value its stability. In case of instability the purpose of the treatment, which depends on the number of levels involved, is to stabilize the segment involved and to promote bone fusion avoiding the development of deformity. It is useful to use the anterior plating for both ACDF or ACCF.

5) Sagittal alignment-kyphosis-lordosis: more levels are involved greater is the probability of developing cervical instability and consequent greater risk to develops a deformity with secondary loss of the physiological lordosis and consequent kyphosis.

Based on our experience and literature results $[5,8,14]$ multilevel ACDF is preferable when compression involves the intersomatic space, or in the early stages of spondylotic myelopaty, when bone degeneration and spinal cord compression are mainly due on intervertebral space. ACDF provides good long term improving in cervical lordosis fusion segment. In addition ACDF restore disc height, promotes posterior ligaments in-buckling correction, maintains a good biomechanical stability during dynamic flexion-extension movements, and maintain a good sagittal alignment. Furthermore, ACDF is less invasive than ACCF in term of blood loss, bone removal, surgical complication (Hoarseness, C5-palsy, dysphagia, dislogment, epidural ematoma, CLF leakage), operation time, hospedalization length. In contrast, if more than 2 levels are involved ACDF can cause pseudoarthrosis or junctional syndrome more likely than ACCF. The pathogenesis and clinical development of adjacent segment degeneration (ASD) are not fully understood. Probably it is due increased stiffness at the fused level, with concomitant increasing in force and motion at adjacent levels or, as Hilibrand and et al. [15] reported it may reflect the natural history of the underlying cervical spondylosis.

Important is the anterior plating [16], required when preoperative instability is present or when more than two contiguous levels are treated $[17,18]$.

ACCF $[9,11,19,20]$ is indicate when spinal cord impingement is behind the vertebral body or when two or three vertebral body are involved or when spondylotic myelopaty is advanced. The surface bone fusion is lower than ACDF, so fusion rate is higher than ACDF if more than 2 or 3 levels are involved. Certainly, it is more invasive than ACDF, it does not restore sagittal alignment and bearing a lesser biomechanical strength of the movements of FE compared to ACDF. In this procedure, the anterior plating by placing the screws both to the upper and lower vertebral body and to the graft too is required to avoid any translational movements.

\section{Conclusions}

There were no significant differences between ACDF and ACCF in clinical outcome at 6 years evaluated by VAS and NDI. The rate of fusion at 6 years for 2 levels ACCF was higher than that for 2 levels ACDF but is not statistically significative. Anyway, ACDF seems to be more efficent to correct cervical kyphosis and to restore SA.

Even if, both ACDF and ACCF can restore lordosis, but in multilevel ACDF, lordosis can be achieved and maintained easier than in ACCF. This is due to the multiple points of distraction and fixation in addition to the graft and interbody space shaping. Therefore, we conclude that it is necessary to preoperatively study patients to classify degenerative disease and biomechanics feature, evaluating the number of levels affected by stenosis, neurological status, the characteristics of the LLP and the stability of the cervical segment. All those are useful parameters to guide the surgeon to choose the best anterior approach for cervical spondylosis.

\section{REFERENCES}

[1] P. G. Matz, P. A. Anderson, L. T. Holly, M. W. Groff, R. F. Heary, M. G. Kaiser, P. V. Mummaneni, et al., "The 
Natural History of Cervical Spondylotic Myelopathy," Journal of Neurosurgery: Spine, Vol. 11, No. 2, 2009, pp. 104-111. http://dx.doi.org/10.3171/2009.1.SPINE08716

[2] S. Nurick, "The Pathogenesis of the Spinal Cord Disorder Associated with Cervical Spondylosis,” Brain, Vol. 95, No. 1, 1972, pp. 87-100. http://dx.doi.org/10.1093/brain/95.1.87

[3] V. H. Hillard and R. I. Apfelbaum, "Surgical Management of Cervical Myelopathy: Indications and Techniques for Multilevel Cervical Discectomy,” The Spine Journal, Vol. 6, No. 6, 2006, pp. S242-S251. 2006http://dx.doi.org/10.1016/j.spinee.2006.05.005

[4] K. Hirabayashi, K. Watanabe, K. Wakano, et al., "Expansive Open-Door Laminoplasty for Cervical Spinal Stenotic Myelopathy,” Spine, Vol. 8, No. 7, 1983, pp. 693699.

http://dx.doi.org/10.1097/00007632-198310000-00003

[5] R. D. Rao, K. Gourab and K. S. David, "Operative Treatment of Cervical Spondylotic Myelopathy,” The Journal of Bone \& Joint Surgery, Vol. 88, No. 7, 2006, pp. 16191640. http://dx.doi.org/10.2106/JBJS.F.00014

[6] B. P. Witwer and G. R. Trost, "Cervical Spondylosis: Ventral or Dorsal Surgery," Neurosurgery, Vol. 60, No. 1, 2007, pp. S130-S136.

[7] Q. S. Lin, X. H. Zhou, X. W. Wang, P. Cao, N. Tsai and W. Yuan, "A Comparison of Anterior Cervical Discectomy and Corpectomy in Patients with Multilevel Cervical Spondylotic Myelopathy,” European Spine Journal, Vol. 21, No. 3, 2012, pp. 474-481.

http://dx.doi.org/10.1007/s00586-011-1961-9

[8] T. J. Stewart, R. P. Schlenk and E. C. Benzel, "Multiple Level Discectomy and Fusion,” Neurosurgery, Vol. 60, No. 1, 2007, pp. S143-S148.

[9] S. Chibbaro, L. Benvenuti, S. Carnesecchi, et al., "Anterior Cervical Corpectomy for Cervical Spondylotic Myelopathy: Experience and Surgical Results in a Series of 70 Consecutive Patients," Journal of Clinical Neuroscience, Vol. 13, No. 2, 2006, pp. 233-238. http://dx.doi.org/10.1016/j.jocn.2005.04.011

[10] S.-D. Jiang, L.-S. Jiang and L.-Y. Dai, “Anterior Cervical Discectomy and Fusion Versus Anterior Cervical Corpectomy and Fusion for Multilevel Cervical Spondylosis: A Systematic Review," Archives of Orthopaedic and Trauma Surgery, Vol. 132, No. 2, 2012, pp. 155-161.

[11] K. Sevki, T. Mehmet, T. Ufuk, et al., "Results of Surgical Treatment for Degenerative Cervical Myelopathy: Anterior Cervical Corpectomy and Stabilization,” Spine, Vol. 29, No. 22, 2004, pp. 2493-2500. http://dx.doi.org/10.1097/01.brs.0000145412.93407.c3

[12] K. Yonenobu, T. Fuji, K. Ono, et al., “Choice of Surgical Treatment for Multisegmental Cervical Spondylotic Myelopathy," Spine, Vol. 10, No. 8, 1985, pp. 710-716. http://dx.doi.org/10.1097/00007632-198510000-00004

[13] D. Konya, S. Ozgen, A. Gercek, et al., "Outcome for Combined Anterior and Posterior Surgical Approaches for Patients with Multisegmental Cervical Spondylotic Myelopathy," Journal of Clinical Neuroscience, Vol. 16, No. 3, 2009, pp. 404-409. http://dx.doi.org/10.1016/j.jocn.2008.07.070

[14] M. Kawakami, T. Tamaki, H. Iwasaki, et al., “A Comparative Study of Surgical Approaches for Cervical Compressive Myelopathy,” Clinical Orthopaedics and Related Research, No. 381, 2000, pp. 129-136. http://dx.doi.org/10.1097/00003086-200012000-00016

[15] A. S. Hilibrand, G. D. Carlson, M. A. Palumbo, P. K. Jones and H. H. Bohlman, "Radiculopathy and Myelopathy at Segments Adjacent to the Site of a Previous Anterior Cervical Arthrodesis," The Journal of Bone \& Joint Surgery, Vol. 81A, No. 4, 1999, pp. 519-28.

[16] A. R. Vaccaro, S. P. Falatyn, G. J. Scuderi, et al. "Early Failure of Long Segment Anterior Cervical Plate Fixation,” Journal of Spinal Disorders, Vol. 11, No. 5, 1998, pp. 410-415.

http://dx.doi.org/10.1097/00002517-199810000-00008

[17] S. W. Chang, U. K. Kakarla, P. H. Maughan, et al., "Four-Level Anterior Cervical Discectomy and Fusion with Plate Fixation: Radiographic and Clinical Results," Neurosurgery, Vol. 66, No. 4, 2010, pp. 639-647. http://dx.doi.org/10.1227/01.NEU.0000367449.60796.94

[18] T. S. Ellison, M. B. Hartman and C. D. Brigham, "Fusion Rates for Two-Level ACDF? Plating versus Subtotal Corpectomy and Fusion," The Annual Meeting of the North American Spine Society, New York, 25 October 1997.

[19] M. Ikenaga, J. Shikata and C. Tanaka, "Long-Term Results over 10 Years of Anterior Corpectomy and Fusion for Multilevel Cervical Myelopathy,” Spine, Vol. 31, No. 14, 2006, pp. 1568-1574. http://dx.doi.org/10.1097/01.brs.0000221985.37468.0f

[20] M. Hussain, A. Nassr, R. N. Natarajan, H. S. An and G. B. J. Andersson, "Corpectomy versus Discectomy for the Treatment of Multilevel Cervical Spine Pathology: A Finite Element Model Analysis,” The Spine Journal, Vol. 12, No. 5, 2012, pp. 401-408. http://dx.doi.org/10.1016/j.spinee.2012.03.025 